\title{
Magnetic resonance quantitative susceptibility mapping in the evaluation of hepatic fibrosis in chronic liver disease: a feasibility study
}

\author{
Zheng Qu ${ }^{1 \#}$, Shuohui Yang ${ }^{2 \#}$, Feng Xing ${ }^{3 \#}$, Rui Tong ${ }^{1}$, Chenyao Yang ${ }^{2}$, Rongfang Guo ${ }^{2}$, Jiling Huang ${ }^{2}$, \\ Fang $\mathrm{Lu}^{2}$, Caixia Fu ${ }^{4}, \mathrm{Xu} \mathrm{\text {Yan}^{4 } \text { , Stefanie Hectors }}{ }^{5}$, Kelly Gillen ${ }^{5}$, Yi Wang, ${ }^{5,6}$ Chenghai Liu ${ }^{3,7}$, \\ Songhua Zhan ${ }^{2}$, Jianqi Li ${ }^{1}$
}

${ }^{1}$ Shanghai Key Laboratory of Magnetic Resonance, School of Physics and Electronic Science, East China Normal University, Shanghai, China; ${ }^{2}$ Department of Radiology, Shuguang Hospital Affiliated to Shanghai University of Traditional Chinese Medicine, Shanghai, China; ${ }^{3}$ Department of Liver Diseases, Shuguang Hospital Affiliated to Shanghai University of Traditional Chinese Medicine, Shanghai, China; ${ }^{4} \mathrm{MR}$ Collaboration NE Asia, Siemens Healthcare, Shanghai, China; ${ }^{5}$ Department of Radiology, Weill Medical College of Cornell University, New York, NY, USA; ${ }^{6}$ Department of Biomedical Engineering, Cornell University, Ithaca, NY, USA; ${ }^{7}$ Shanghai Key Laboratory of Traditional Chinese Clinical Medicine, Shanghai, China

"These authors contributed equally to this work.

Correspondence to: Chenghai Liu, PhD, MD. Department of Liver Diseases, Shuguang Hospital Affiliated to Shanghai University of Traditional Chinese Medicine, 528 Zhangheng Road, Shanghai 201203, China. Email: chenghai.liu@outlook.com; Songhua Zhan PhD, MD. Department of Radiology, Shuguang Hospital Affiliated to Shanghai University of Traditional Chinese Medicine, 528 Zhangheng Road, Shanghai 201203, China. Email: zhansonghua@sina.com; Jianqi Li, PhD. Shanghai Key Laboratory of Magnetic Resonance, School of Physics and Electronic Science, East China Normal University, 3663 North Zhongshan Road, Shanghai 200062, China. Email: jqli@phy.ecnu.edu.cn.

Background: Noninvasive methods for the early diagnosis and staging of hepatic fibrosis are needed. The present study aimed to investigate the alteration of magnetic susceptibility in the liver of patients with various fibrosis stages and to evaluate the feasibility of using susceptibility to stage hepatic fibrosis.

Methods: A total of 30 consecutive patients with chronic liver diseases (CLDs) underwent magnetic resonance imaging (MRI) and liver biopsy evaluation of hepatic fibrosis, necroinflammatory activity, iron load, and steatosis. Quantitative susceptibility mapping (QSM), R2* and proton density fat fraction (PDFF) images were postprocessed from the same gradient-echo data for quantitative tissue characterization using region of interest (ROI) analysis. The differences for MRI measurements between cohorts of non-significant (Ishak-F <3) and significant fibrosis (Ishak-F $\geq 3$ ) and the correlation of MRI measurements with fibrosis stages and necroinflammatory activity grades were tested. Receiver operating characteristic (ROC) analysis was also performed.

Results: There was a significant difference in liver susceptibility between the cohorts of significant and non-significant fibrosis $(\mathrm{Z}=-2.880, \mathrm{P}=0.004)$. A moderate negative correlation between the stages of liver fibrosis and liver susceptibility was observed $(r=-0.471, \mathrm{P}=0.015)$. Liver magnetic susceptibility differentiated non-significant from significant hepatic fibrosis with an area under the receiver operating curve (AUC) of $0.836(\mathrm{P}=0.004)$. A highly sensitive diagnostic performance with an AUC of 0.933 was obtained using magnetic susceptibility and PDFF together $(\mathrm{P}<0.001)$.

Conclusions: A noninvasive liver QSM-based evaluation promises an accurate assessment of significant fibrosis in patients with CLDs.

Keywords: Chronic liver disease (CLD); fibrosis; liver; quantitative susceptibility mapping (QSM)

Submitted Jun 01, 2020. Accepted for publication Oct 31, 2020.

doi: 10.21037/qims-20-720

View this article at: http://dx.doi.org/10.21037/qims-20-720 


\section{Introduction}

Hepatic fibrosis is a wound-healing response to repeated damage to the liver caused by multiple pathogenic factors including viral, autoimmune, drug-induced, cholestatic, and metabolic diseases (1). Hepatic fibrosis, a pathological progression common among most types of chronic liver diseases (CLDs), can result in cirrhosis, progressive hepatic dysfunction, portal hypertension, and in some cases, hepatocellular carcinoma (2). Hepatic cirrhosis is an irreversible process, but hepatic fibrosis progression is potentially reversible through drug therapy based on a careful and accurate evaluation of the extent of the hepatic fibrosis. Therefore, with the number of patients suffering from CLDs continually increasing, early diagnosis and staging of hepatic fibrosis have significant clinical value for patient management decisions, treatment stratification, and prognostication.

Pathological assessment via liver biopsy is the current gold standard for staging hepatic fibrosis. However, this invasive procedure is risky because of potential complications including hemorrhage, wound infection, and perforation of other organs. Moreover, liver biopsy is known to suffer from sampling errors due to fibrosis heterogeneity (3). Therefore, the exploration of a reliable, reproducible, and noninvasive alternative for detection and accurate staging of hepatic fibrosis is a highly relevant research topic.

With their excellent resolution and soft tissue contrast, various magnetic resonance imaging (MRI) techniques have been proposed for the noninvasive diagnosis and staging of hepatic fibrosis, including diffusion-weighted imaging (DWI) (4), MR perfusion imaging (5), dynamic contrast-enhanced MRI (6), spin-lattice relaxation time in the rotating frame (T1rho) MRI $(7,8)$, susceptibilityweighted imaging (SWI) (9), MR elastography (MRE) (10), and multiparametric MRI quantitative techniques $(11,12)$. However, the apparent diffusion coefficient (ADC) value measured by DWI could be affected by steatosis and iron overload (13). MR perfusion imaging in the liver is complicated by the effects of a dual blood supply and respiratory artifacts (5), while dynamic contrast-enhanced MR imaging may carry a risk to patients with renal insufficiency because of the injected [Gd] contrast agent. T1rho MR imaging is rather sensitive to magnetic field inhomogeneity, and this technique requires higher energy deposition produced by radio frequency (RF). SWI is a nonquantitative method with contrasts dependent on imaging parameters, and the tissue signal intensity ratio is dependent on the sequence provided by different MRI scanners. MRE is advantageous over ultrasound elastography and other MRI-based techniques because of its accuracy and reliability for diagnosing and grading liver fibrosis; however, the requirement for costly additional equipment limits its accessibility and application (13). Meanwhile, a multiparametric imaging approach to the liver may suffer from misregistration errors, because the images of multiple contrasts have to be acquired with multiple breath-holds.

Quantitative susceptibility mapping (QSM) is a postprocessing technique that can accurately provide the quantitative distribution of susceptibility sources in tissue (14). QSM cleans up the acquired, but often discarded, phase information of the widely available gradient echo (GRE) sequence (15) and performs the fieldto-source ill-posed inversion using Bayesian inference (16). QSM has been applied to abdominal organs by accounting for the fat contribution to the measured signal phase $(17,18)$. QSM reproducibility has been demonstrated in the brain (19-23), lumbar vertebra (24), and liver $(25,26)$. Hepatic fibrosis is characterized by excessive accumulation of extracellular matrix (ECM) proteins including collagen $(1,3)$, which can contribute to strong diamagnetic susceptibility in the liver tissue due to the enhanced density of orbiting electrons (27). A previous in-vitro QSM study demonstrated that susceptibility was linearly dependent on collagen concentration (28). Therefore, QSM provides a method for measuring collagen to directly reflect the degree of fibrosis and should be investigated as a feasible tool for the noninvasive staging of hepatic fibrosis.

Thus, the purpose of this study was to investigate changes in liver magnetic susceptibility in patients with various stages of fibrosis, and to evaluate the feasibility of using susceptibility to stage hepatic fibrosis.

\section{Methods}

\section{Human subjects}

This prospective study was conducted after obtaining approval from the Institutional Review Board of Shuguang Hospital affiliated with Shanghai University of Traditional Chinese Medicine (approval number: 2018-625-54-01), and written informed consent was obtained from each participant. Thirty consecutive patients with suspected or known CLDs who were willing to take an MRI-QSM 


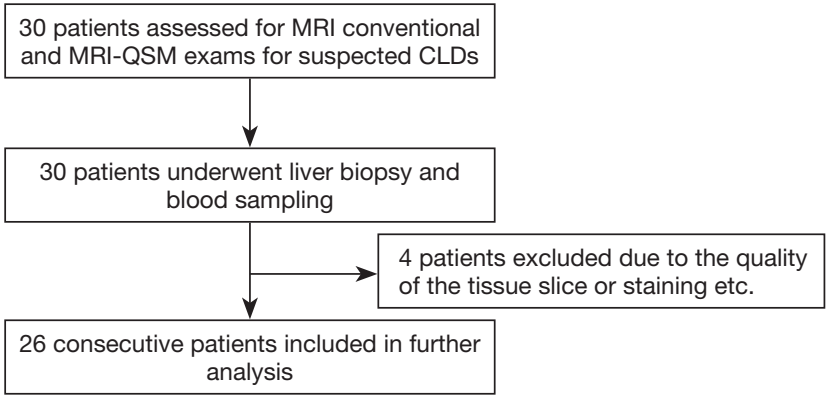

Figure 1 Flowchart showing participant recruitment. CLD, chronic liver disease; QSM, quantitative susceptibility mapping.

examination and biopsy were recruited for this study. MRI was performed before the liver biopsy. The interval between the MRI examinations, biopsy, and blood sampling was within 2 days. Four of the patients were excluded due to the poor quality of the tissue slice and staining. The final cohort consisted of 26 patients. Figure 1 summarizes the patient recruitment in this study.

\section{MR imaging}

The patients were imaged on a clinical 3T MRI system (Skyra; Siemens Healthcare, Erlangen, Germany) using an 18-channel body coil in combination with some elements of the spine coil. To obtain the susceptibility and R2* maps, complex data were acquired by using a $3 \mathrm{D}$ monopolar readout gradient volumetric interpolated breathhold examination (VIBE) sequence with the following parameters: repetition time $(\mathrm{TR})=11.3 \mathrm{~ms}$, flip angle $=4^{\circ}$, echo number $=6$, the first echo time $\left(\mathrm{TE}_{1}\right)=1.07 \mathrm{~ms}$, echo spacing $=1.79 \mathrm{~ms}$, bandwidth $=1,060 \mathrm{~Hz} /$ pixel, field of view $(\mathrm{FOV})=400 \times 350 \mathrm{~mm}^{2}$, matrix size $=224 \times 196 \times 52$, voxel size $=1.8 \times 1.8 \times 3.5 \mathrm{~mm}^{3}$. Additionally, a CAIPIRINHA parallel imaging technique with an acceleration factor of $2 \times 2$ and $6 / 8$ fractional Fourier transform in the phase-encoding direction was used to reduce acquisition time. The acquisition time for each sequence was 17 seconds during one breath hold. Conventional MR images, including T1and $\mathrm{T} 2$-weighted images, were also acquired for anatomical evaluation.

\section{Image processing}

Figure 2 is a schematic of the QSM reconstruction. Phase images and magnitude images were extracted from the complex MRI data. Simultaneous phase unwrapping and removal of chemical shift (SPURS) (29) using graph cuts with conditional jump moves were first performed on the phase images, followed by fine-tuning of the field map with T2*-IDEAL with a single R2* and 6-peak fat model for the combined water-fat signal (30). The output inhomogeneity B0 field of T2*-IDEAL was then processed with background field removal using the projection onto dipole field (PDF) method (31), and the remaining magnetic field was processed to generate a susceptibility map using the morphology enabled dipole inversion algorithm (MEDI) (32) with the regularization parameter lambda set to 1,000 and the parameter SMV set to $5 \mathrm{~mm}$.

The proton density fat fraction (PDFF) image was generated from the obtained fat and water images. PDFF was defined by the following equation:

$$
\text { PDFF }=\frac{\rho_{\text {fat }}}{\rho_{\text {fat }}+\rho_{\text {water }}}
$$

where $\rho_{\text {fat }}$ and $\rho_{\text {water }}$ are the signal amplitude of the fat image and water image, respectively.

The data processing was performed using Matlab2016b (MathWorks, Natick, MA, USA).

\section{Image analysis}

The image quality of the QSM images was assessed independently by two radiologists (with 3 and 12 years' experience in hepatic MRI) based on the radiological impression of image blurring, deformity, and other artifacts, and scored with the following 4 point scale: 0 , poor image quality with serious artifacts; 1 , diagnostic with moderate image quality and substantial artifacts; 2, diagnostic with good image quality and marginal artifacts; and 3, diagnostic with excellent image quality and no artifacts. Only QSM images with acceptable image quality scores $(\geq 1)$ were used for the analyses.

Liver magnetic susceptibility, R2*, and PDFF values were measured using region of interest (ROI) analysis. ROIs were manually drawn on the QSM images using ITK-SNAP software (version 3.8.0, www.itksnap.org) by the two radiologists mentioned above who were blinded to the clinical history, blood markers data, and liver biopsy results. First, through consensus, both reviewers determined the single liver slice which contained an aslarge-as-possible cross section through the liver and the least motion artifacts to measure. Then, ROIs ranging from 5 to $8 \mathrm{~cm}^{2}$ were independently placed in this consensus slice in the anterior and posterior segment of the right lobe of 


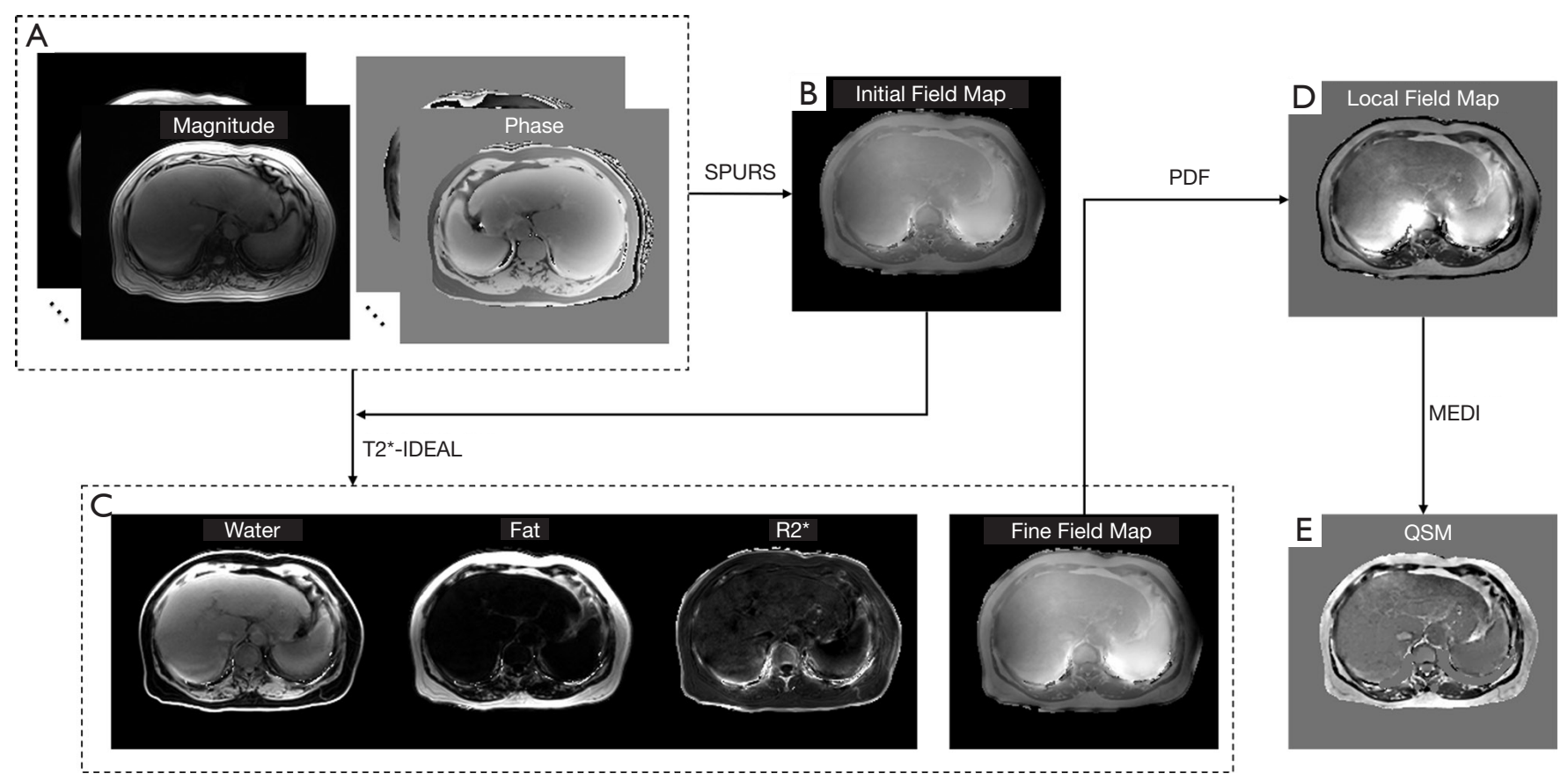

Figure 2 The schematic view of liver QSM reconstruction. SPURS is performed on the magnitude and phase images (A) to initialize the inhomogeneity magnetic field (B). Then, the T2*-IDEAL algorithm is performed, outputting water, fat, R2*, and fine field maps (C). The fine field maps are then processed with background field removal to obtain the local magnetic field maps (D) using the PDF method. The remaining magnetic field maps are processed to generate a susceptibility map (E) by MEDI. QSM, quantitative susceptibility mapping; SPURS, simultaneous phase unwrapping and removal of chemical shift; IDEAL, iterative decomposition of water and fat with echo asymmetry and least-squares estimation; PDF, projection onto dipole field; MEDI, morphology enabled dipole inversion algorithm.

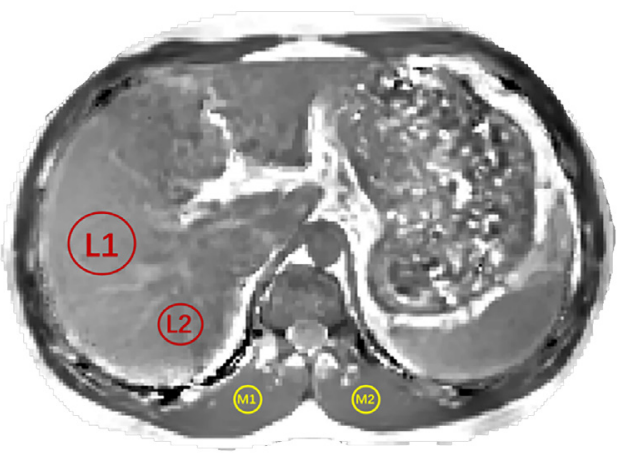

Figure 3 The ROIs for QSM, R2*, and PDFF value measurements. ROI analyses were performed at two locations (anterior and posterior segment of the right lobe) in the liver (L1, L2) and at two locations in the latissimus dorsi muscle (M1, M2). To select a region in which the signal was as homogeneous as possible and to avoid large blood vessels and cavities, L1 was made larger than L2. ROI, regions of interest; QSM, quantitative susceptibility mapping; PDFF, proton density fat fraction. the liver where a biopsy specimen was obtained (Figure 3). The other two ROIs ranging from 1.8 to $3.6 \mathrm{~cm}^{2}$ in the same slice were placed in the latissimus dorsi muscle on either side of the spine as a reference for determining the relative susceptibility in the hepatic parenchyma, as the latissimus dorsi muscle does not accumulate iron $(28,33)$. All ROIs were drawn in the area in which the signal was as homogeneous as possible, and large blood vessels and cavities were avoided. ROIs in the liver were at least $7.2 \mathrm{~mm}$ away from the air boundary, while ROIs in the latissimus dorsi muscle were 3.6-7.2 $\mathrm{mm}$ away from the tissue border and the air boundary. The ROIs were then copied to corresponding R2* and PDFF images for measurements. $\mathrm{R}^{*}$ and PDFF values were measured and averaged on the liver ROIs, while the relative value between the averaged values on the liver ROIs and on the latissimus dorsi muscle ROIs was calculated as the hepatic susceptibility value. To evaluate the reproducibility of the ROI sampling, the above ROI drawing and analyses were repeated by both radiologists after 4 months. 
Table 1 Ishak fibrosis scoring system

Score stage description

0 No fibrosis

1 Fibrous expansion of some portal areas, with or without short fibrous septa

2 Fibrous expansion of most portal areas, with or without short fibrous septa

3 Fibrous expansion of most portal areas with occasional portal to portal $(\mathrm{P}-\mathrm{P})$ bridging

4 Fibrous expansion of portal areas with marked bridging [portal to portal (P-P) and portal to central (P-C)]

5 Marked bridging (P-P and/or P-C) with occasional nodules (incomplete cirrhosis)

6 Cirrhosis, probable, or definite

\section{Blood markers and histopathologic evaluations}

Serum markers including serum alanine aminotransferase (ALT, units per liter), serum aspartate aminotransferase (AST, units per liter), g-glutamyl transpeptidase (GGT, units per liter), serum albumin (Alb, grams per liter), total serum bilirubin (Tbil, micromoles per liter), and the ASTALT ratio were used to assess liver function.

The liver biopsy specimens were obtained by an associate physician in the Institute of Liver Diseases using an automatic needle device (MAGNUM biopsy instrument, Bard, USA) with a 16-gauge disposable needle (MN1620, Bard, USA). Pathological samples of around $1 \mathrm{~mm}$ in diameter and $17 \mathrm{~mm}$ in length were fixed with $10 \%$ formalin, embedded in paraffin, and sectioned. The tissue slices were stained with hematoxylin-eosin (HE), Masson's trichrome, reticulin, cytokeratin (CK) 7, CK19, CD34, CD8, hepatitis B surface antigen ( $\mathrm{HBsAg}$ ), hepatitis B core antigen ( $\mathrm{HBcAg}$ ), and Perls' Prussian blue.

Hepatic fibrosis was evaluated semiquantitatively with the modified Ishak histological activity index scale ranging from 0-6 based on the reticulin and Masson's trichrome staining (34). A detailed scoring description is shown in Table 1. The recent guidelines of the American Association for the Study of Liver Diseases (AASLD) and the European Association for the Study of Liver (EASL) both recommended antiviral therapy for patients with evidence of significant fibrosis (Ishak fibrosis score $\geq 3$ ) $(35,36)$, so we performed further quantitative measurements based on this classification: significant fibrosis (Ishak fibrosis score $\geq 3$ ) and non-significant fibrosis (Ishak fibrosis score $<3$ ).
Necroinflammatory activity was evaluated semiquantitatively according to the METAVIR scoring system (37) as follows: 0 , none; 1 , mild; 2, moderate; and 3, severe. According to the proportion of hepatocytes containing fat vesicles based on HE staining, liver steatosis was graded as follows: none, $<5 \%$; slight, 5-33\%; moderate, 33-66\%; and severe, $>66 \%$. Iron overload was classified as positive or negative based on the visual assessment of iron granules with Prussian blue stain (38).

All the liver biopsies were graded by three hepatologists independently, and the final scores were obtained by consensus from at least two hepatologists. If the three hepatologists had different results, liver biopsy samples were evaluated and decided by the central hepatologist.

\section{Statistical analysis}

Grading scales of the image quality are expressed as median [P25, P75], and differences between the two reviewers were evaluated with Wilcoxon signed-rank test. Sex and histopathologic grading are presented as numbers, whereas descriptive statistics of all other variables are expressed as medians and confidence intervals. The difference for anthropometric, blood markers, and MRI measurements between cohorts of non-significant (Ishak-F <3) and significant (Ishak-F $\geq 3$ ) fibrosis were tested by using the Mann-Whitney $\mathrm{U}$ test. For the MRI measurements that were significantly different between the two cohorts, the distribution for each cohort is shown with box plots. A nonparametric Spearman's rank correlation test was performed to evaluate the correlation of MRI measurements with fibrosis stages and necroinflammatory activity grades. Receiver operating characteristic (ROC) analysis was performed based on binary logistic regression to assess the diagnostic utility of MRI measurements (liver susceptibility, R2*, PDFF, and their combinations) to differentiate non-significant from significant fibrosis. The value that maximized the Youden index was chosen as the optimal cutoff value. To evaluate pathological distribution characteristics, Kendall's tau-b coefficient was calculated to measure the degree of correspondence between the fibrosis stage and other histopathologic grades (necroinflammatory activity grade and liver steatosis extent).

To evaluate intra- and interobserver reproducibility, reliability analysis was performed by calculating the intraclass correlation coefficients (ICC) based on the QSM ROI measurements.

All statistical tests were performed using SPSS software (version 23.0; SPSS, Chicago, IL, USA) and OriginPro software (version 8.0; OriginLab Corporation, MA, USA). 
Table 2 Comparison of the anthropometric measurements and blood markers between non-significant (Ishak- $\mathrm{F}<3$ ) and significant (Ishak- $\mathrm{F} \geq 3$ ) fibrosis groups

\begin{tabular}{lccc}
\hline Parameters & All subjects $(n=26)$ & Ishak-F $<3(n=11)$ & Ishak-F $\geq 3(n=15)$ \\
\hline Age (years) & $48.00(43.22,52.93)$ & $41.00(37.17,55.19)$ & $48.00(43.28,55.66)$ \\
BMI $\left(\mathrm{kg} / \mathrm{m}^{2}\right)$ & $23.95(22.18,25.31)$ & $23.30(20.03,26.81)$ & $24.20(22.37,25.61)$ \\
Sex & & & 0.377 \\
Male & 17 & 8 & 9.640 \\
Female & 9 & 3 & 6 \\
ALT (U/L) & $40.00(42.40,132.75)$ & $34.00(19.86,124.14)$ & $41.00(25.31,172.69)$ \\
AST (U/L) & $33.50(32.78,84.43)$ & $43.68(15.90,80.43)$ & $36.00(25.18,107.35)$ \\
GGT (U/L) & $57.00(35.79,176.57)$ & $48.00(-30.61,302.12)$ & 0.678 \\
Alb (g/L) & $41.95(39.92,43.49)$ & $42.90(39.36,46.02)$ & 0.194 \\
Tbil $(\mu \mathrm{mol} / \mathrm{L})$ & $17.48(15.46,22.94)$ & $15.20(11.98,24.46)$ & $41.30(38.77,43.18)$ \\
AST/ALT & $0.85(0.74,1.02)$ & $0.67(0.58,1.07)$ & $17.66(14.66,25.18)$ \\
\hline
\end{tabular}

Sex is presented as numbers, while other data are expressed as medians and 95\% confidence intervals. ALT, alanine aminotransferase; AST, aspartate aminotransferase; GGT, g-glutamyl transpeptidase; Alb, albumin; Tbil, total serum bilirubin.

A P value $<0.05$ was considered statistically significant.

\section{Results}

\section{Patient characteristics}

The final cohort consisted of 26 patients (mean age, $48.08 \pm 12.03$ years; range, $27-68$ years) diagnosed with CLDs on the basis of previous clinical, blood marker exam results, and MRI. These patients, including 17 men (mean age, $44.82 \pm 10.82$ years; range, $27-64$ years) and 9 women (mean age, 54.22 \pm 12.37 ; range, 33-68 years), had the following CLDs: chronic hepatitis $B(n=9,34.6 \%)$, cryptogenic hepatic fibrosis $(\mathrm{n}=5,19.2 \%)$, hepatic injury $(\mathrm{n}=2,7.7 \%)$, steatohepatitis $(\mathrm{n}=1,3.8 \%)$, steatohepatitis with chronic hepatitis $B(n=2,7.7 \%)$, steatohepatitis with autoimmune hepatitis with $(\mathrm{n}=1,3.8 \%)$, alcoholic hepatic disease $(n=1,3.8 \%)$, alcoholic hepatic disease with schistosome $(n=1,3.8 \%)$, chronic hepatitis with autoimmune hepatitis $(\mathrm{n}=1,3.8 \%)$, primary biliary cirrhosis $(\mathrm{n}=1,3.8 \%)$, autoimmune hepatitis $(\mathrm{n}=1,3.8 \%)$, and primary biliary cirrhosis with hepatic injury $(\mathrm{n}=1,3.8 \%)$.

\section{Anthropometric measurements and blood markers}

According to the Ishak fibrosis scores, 15 patients (58\%) had significant hepatic fibrosis (Ishak-F $\geq 3$ ) and 11 patients
$(42 \%)$ had non-significant hepatic fibrosis (Ishak-F $<3$ ). Anthropometric measurements, blood markers, and imaging results are summarized in Table 2. There were no statistically significant differences between the two fibrosis cohorts in age, BMI, gender distribution, or blood markers.

\section{QSM, R2*, and PDFF measurements}

The image quality scores for QSM were diagnostic for all patients, and thus all images could be used for further analysis. No significant difference in image quality was found between reviewer $1[2.00(1.00,2.25)]$ and reviewer 2 $[2.00(1.00,2.25)](Z=-0.277, \mathrm{P}=0.782)$.

The results of the MRI measurements are summarized in Table 3. A significant difference in liver susceptibility was observed between the two patient cohorts $(Z=-2.880$, $\mathrm{P}=0.004)$. The mean susceptibility value of the significant fibrosis cohort was $0.1051 \pm 0.0663 \mathrm{ppm}$, which was significantly lower than that of the non-significant fibrosis cohort $(0.1971 \pm 0.0748 \mathrm{ppm})$. The susceptibility values between the two cohorts are also illustrated with boxplots in Figure 4. There were no significant differences in R2* and PDFF values between the two cohorts. Only two of the patients in this study had a PDFF value over $5.56 \%$, which was taken as the cutoff value for hepatic steatosis.

The results of the nonparametric Spearman's rank correlation test of the MRI measurements with the 
Table 3 Comparison of the MRI measurements in patients between non-significant (Ishak-F <3) and significant (Ishak-F $\geq 3$ ) fibrosis groups

\begin{tabular}{lllll}
\hline Parameters & All subjects $(\mathrm{n}=26)$ & Ishak $-\mathrm{F}<3(\mathrm{n}=11)$ & Ishak- $\mathrm{F} \geq 3(\mathrm{n}=15)$ & $\mathrm{P}$ \\
\hline$\chi_{\text {liver }}(\mathrm{ppm})$ & $0.1471(0.1106,0.774)$ & $0.1746(0.1469,0.2473)$ & $0.1170(0.0684,0.1418)$ & 0.004 \\
$\mathrm{R}^{*}\left(\mathrm{~s}^{-1}\right)$ & $44.99(40.60,52.88)$ & $45.30(38.04,56.15)$ & $44.67(37.16,55.79)$ & 0.815 \\
PDFF & $2.78 \%(2.40 \%, 5.00 \%)$ & $1.90 \%(1.58 \%, 3.28 \%)$ & $4.30 \%(2.43 \%, 6.76 \%)$ & 0.102 \\
\hline
\end{tabular}

Data are expressed as medians and $95 \%$ confidence intervals. $\chi_{\text {liver }}$, live susceptibility; PDFF, proton density fat fraction.

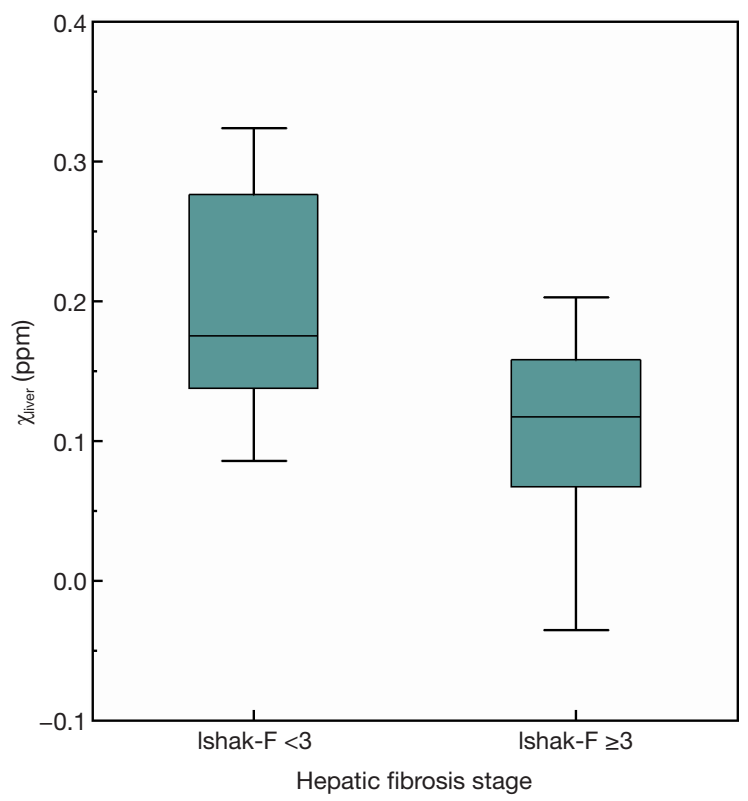

Figure 4 Boxplot analysis of the liver susceptibility value between cohorts of Ishak-F $<3$ and Ishak-F $\geq 3$. The susceptibility value decreased significantly with increasing fibrosis stage.

Table 4 The correlation analyses of MRI measurements with the fibrosis stage and the necroinflammatory activity grade

\begin{tabular}{lll}
\hline Measurements & Fibrosis stage & $\begin{array}{l}\text { Necroinflammatory } \\
\text { activity grade }\end{array}$ \\
\hline$\chi_{\text {liver }}(\mathrm{ppm})$ & $\mathrm{r}=-0.471$ & $\mathrm{r}=-0.168$ \\
& $\mathrm{P}=0.015$ & $\mathrm{P}=0.412$ \\
$\mathrm{R}^{*}\left(\mathrm{~s}^{-1}\right)$ & $\mathrm{r}=-0.001$ & $\mathrm{r}=0.010$ \\
& $\mathrm{P}=0.996$ & $\mathrm{P}=0.961$ \\
PDFF & $\mathrm{r}=0.272$ & $\mathrm{r}=0.278$ \\
& $\mathrm{P}=0.178$ & $\mathrm{P}=0.168$ \\
\hline
\end{tabular}

$r$ is Spearman's rank correlation coefficient. $\chi_{\text {liver }}$, live susceptibility; PDFF, proton density fat fraction.

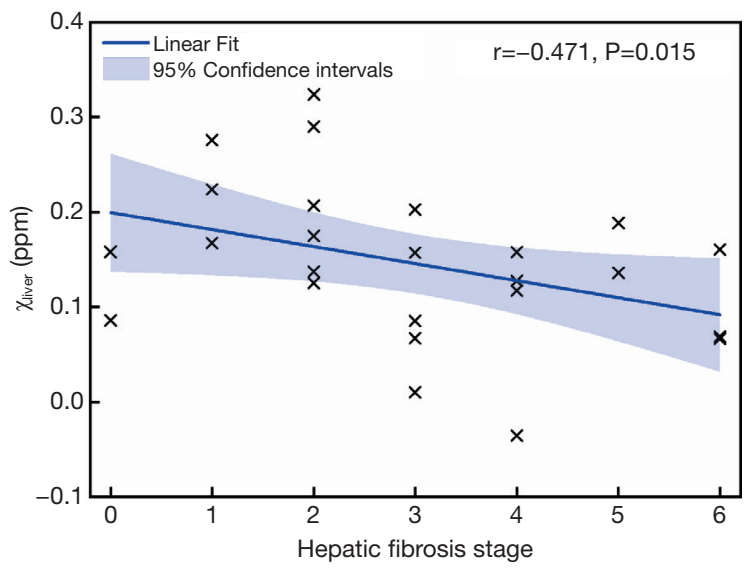

Figure 5 Correlation analysis of liver susceptibility values with the Ishak fibrosis scores. The susceptibility values are moderately and negatively correlated with the fibrosis stage. The correlation coefficient was calculated using the Spearman's rank correlation test.

fibrosis stages and necroinflammatory activity grades are summarized in Table 4. There was a moderate negative correlation between the susceptibility value and the fibrosis stage of patients $(r=-0.471, \mathrm{P}=0.015)$, which is also shown in Figure 5. However, there was no significant correlation of $\mathrm{R} 2$ * or PDFF with the fibrosis stage. No significant correlations of the MRI measurements with the necroinflammatory activity grade were observed.

\section{ROC analysis}

Figure 6 shows the ROC curves of the MRI measurements (liver susceptibility, R2*, PDFF, and their combinations) for differentiating significant from non-significant hepatic fibrosis. The areas under the curve (AUC), asymptotic significance, and asymptotic $95 \%$ confidence intervals of the MRI measurements for differentiating significant from non-significant hepatic fibrosis are summarized in Table 5. The ROC curves demonstrated that liver susceptibility had a superior ability to differentiate non-significant from 
significant hepatic fibrosis ( $\mathrm{AUC}=0.836, \mathrm{P}=0.004$ ) over $\mathrm{R} 2$ * $(\mathrm{AUC}=0.572, \mathrm{P}=0.815)$ and $\mathrm{PDFF}(\mathrm{AUC}=0.691, \mathrm{P}=0.102)$. The optimal cutoff value of susceptibility for non-significant versus significant hepatic fibrosis was $\leq 0.1639 \mathrm{ppm}$ with the Youden index as 0.503. A better diagnostic performance was obtained using susceptibility and PDFF together (AUC $=0.933, \mathrm{P}<0.001)$.

\section{Pathological distribution characteristics}

The distribution of patients in each hepatic fibrosis stage,

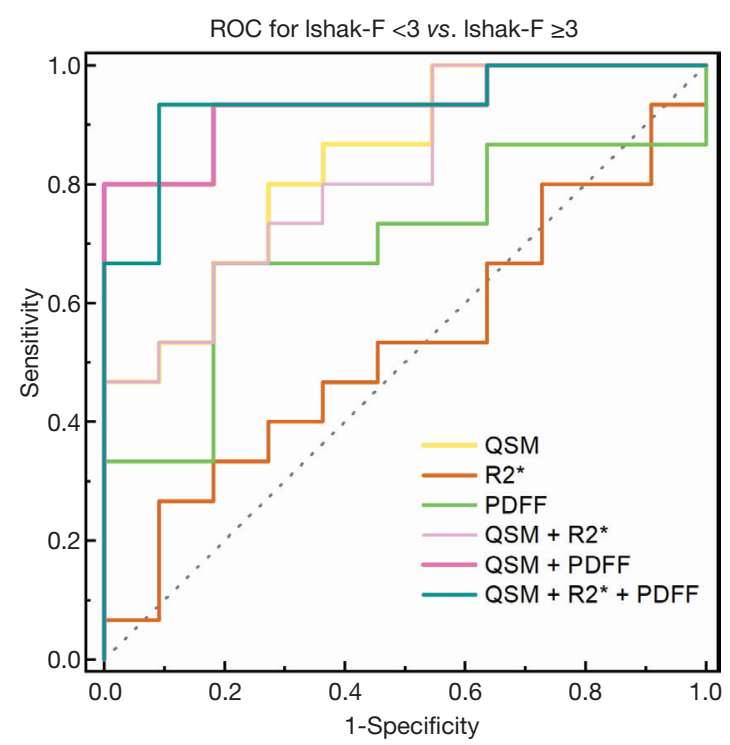

Figure 6 The ROC curves of the MRI measurements (liver susceptibility, R2*, PDFF, and their combinations) for differentiating significant from non-significant hepatic fibrosis. ROC, receiver operating characteristic; PDFF, proton density fat fraction necroinflammatory activity grade, steatosis, and iron load degree is summarized in Table 6. Kendall's tau-b rank correlation test revealed that the fibrosis stage correlated with an increasing necroinflammatory activity grade $(r=0.664, \mathrm{P}<0.001)$. However, there was no significant correlation between the fibrosis stage and the degree of steatosis in hepatocytes $(r=0.251, \mathrm{P}=0.139)$.

\section{Inter- and Intra-observer reliability}

Interobserver reliability between the two reviewers was almost perfect (ICC, 0.977; $\mathrm{P}<0.001 ; 95 \%$ confidence interval: $0.949,0.990)$. The intra-observer ICCs were 0.917 and 0.983 for the two reviewers, demonstrating good reproducibility on the ROI measurements.

\section{Discussion}

Our results demonstrate the clinical feasibility and accurate diagnostic performance of liver QSM for assessing and staging hepatic fibrosis. QSM can noninvasively capture the susceptibility change during the development of hepatic fibrosis in patients with CLDs. Our data show that liver susceptibility had a moderate negative correlation with fibrosis stage, and that liver susceptibility values in patients decreased significantly with significant fibrosis compared to non-significant fibrosis. A highly accurate diagnostic performance was achieved in the cohort of CLD patients by integrating susceptibility and PDFF together. QSM is thus a feasible noninvasive diagnostic tool for differentiating fibrosis stages in patients with CLDs.

The QSM-based diagnostic accuracy in assessing significant liver fibrosis found in this preliminary study (AUC $=0.933)$ has an obvious advantage over the serum biomarkers $(\mathrm{AUC}=0.71-0.81)(39,40)$ and over multiparametric MRI

Table 5 The diagnostic performance of MRI measurements for differentiating significant from non-significant hepatic fibrosis

\begin{tabular}{llcc}
\hline Diagnosis marker & AUC & Asymptotic significance & Asymptotic 95\% confidence intervals \\
\hline QSM & 0.836 & 0.004 & $(0.683,0.989)$ \\
R2* & 0.527 & 0.815 & $(0.301,0.753)$ \\
PDFF & 0.691 & 0.102 & $(0.482,0.900)$ \\
QSM + R2* & 0.818 & 0.006 & $(0.657,0.979)$ \\
QSM + PDFF & 0.933 & $<0.001$ & $(0.837,1.000)$ \\
QSM + R2* + PDFF & 0.933 & $<0.001$ & $(0.834,1.000)$
\end{tabular}

AUC, areas under the curve; QSM, quantitative susceptibility mapping; PDFF, proton density fat fraction. 
Table 6 Distribution of patients in each histopathologic grade

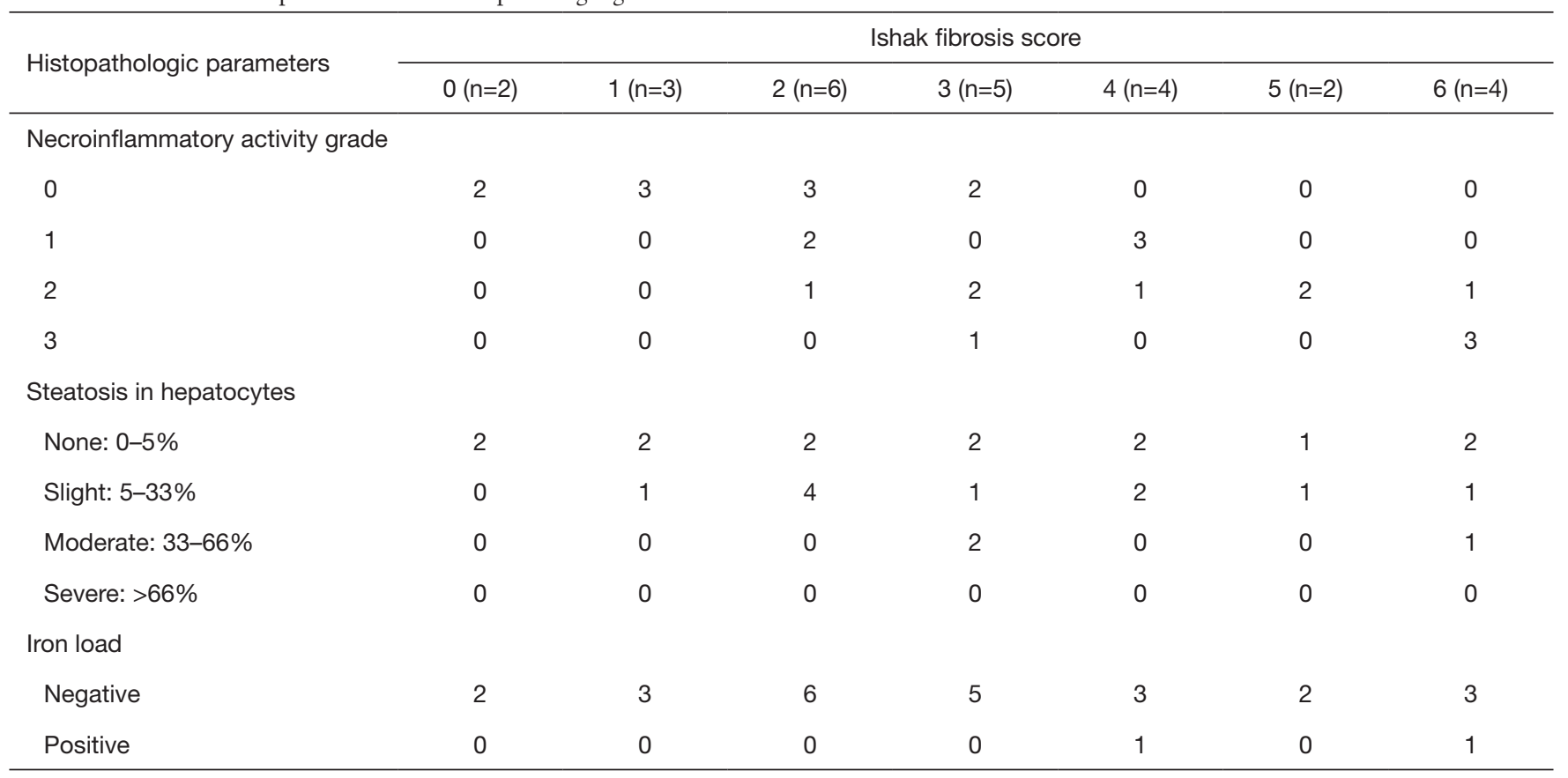

techniques such as FibroMRI (AUC =0.85) (12) and the liver inflammation and fibrosis score (AUC $=0.89)(11)$. Moreover, the diagnostic accuracy of the QSM-based method matches that of MRE (AUC =0.92-0.94) (41-43), which is arguably the most accurate MRI technique for assessing liver fibrosis among available the methods based on diffusion, perfusion, T1rho, or mechanics response (13). QSM-based fibrosis assessment takes the chemical composition approach to evaluating liver fibrosis by logistically integrating magnetic susceptibility, proton density fat fraction, and R2* values all derived from a single, widely available multiecho GRE sequence without any additional hardware requirement. QSM has also been demonstrated to be very reproducible across scanner platforms (19) and readily applicable for imaging the liver (25). In comparison, MRE takes the mechanical approach to evaluating liver fibrosis by probing the tissue with a mechanical wave and measuring its response, necessitating expensive wave driver hardware. A QSM-based approach may be combined with MRE for a more accurate assessment of liver fibrosis, and a QSM-based approach may be used when MRE hardware is not available.

It is well known that multifactorial and often diseasespecific hepatic injury leads to the initiation of fibrogenesis (3). The stimuli followed by activation of the innate immune system provoke a fibrogenic response with ECM-producing myofibroblasts, and liver fibrosis is characterized by excessive accumulation of ECM proteins including collagen $(1,3)$. These proteins have an enhanced density of orbiting electrons and may contribute to strong diamagnetic susceptibility in the tissue (27), as demonstrated in previous studies with in-vitro collagen phantoms (28), articular cartilage specimens (44), and the fibrotic liver tissues (45). We found a moderate negative linear correlation between the measured susceptibility values and the fibrosis stage of the patients in this study, which suggests that an increase in collagen quantity could be reflected by a decrease in susceptibility, with this observed decrease in susceptibility being closely related to the progression of fibrosis.

Liver iron and fat may also contribute to liver tissue susceptibility. Iron overload leads to the accumulation of iron in tissues and tends to follow distinct patterns in which the liver is a common site of deposition, resulting in an increase in tissue susceptibility due to its paramagnetic effects. Lipids are less diamagnetic than water, resulting in an increase in susceptibility (46). In our study, only two of the patients were diagnosed as positive in iron overload through liver biopsy, and half of the patients suffered from slight-to-moderate steatohepatitis. It is unclear what value R2* can add to fibrosis diagnosis by QSM in patients with little iron overloading, and it may be more useful in patients with more substantial liver iron overloading.

Hepatic steatosis existing alone or as a cofactor with 
other CLDs can exacerbate liver injury and make the liver more vulnerable to factors that lead to liver inflammation and fibrosis (47). Meanwhile, increasing evidence has demonstrated that hepatic steatosis is an additional metabolic risk factor of chronic hepatitis B-related fibrosis (46). In our study, $42.3 \%$ of CLD patients suffered from chronic hepatitis $\mathrm{B}$. The histopathologic results revealed that half of the patients suffered from slight-tomoderate steatohepatitis. Although no statistical difference of PDFF was observed between cohorts of non-significant and significant fibrosis, there was a trend that the PDFF value increased with the fibrosis stage, with a mean PDFF value of $1.9 \%$ for patients with non-significant fibrosis and $4.3 \%$ for patients with significant fibrosis. This may explain the increase on the AUC of susceptibility combined with PDFF. Therefore, QSM combined with a PDFF biomarker showed a better diagnostic performance than QSM alone.

The presence of fibrosis, fat, and iron in tissue does not only alter the susceptibility of tissue but also increases the local field inhomogeneity, which further increases the R2* value (48). However, the presence of necroinflammation with fibrosis reduces R2 and R2* because liver inflammation is typically accompanied by cell swelling, edema, and early loss of plasma membrane integrity with an accumulation of inflammatory necrotic tissue (49). No significant correlations of liver R2* with the fibrosis stage or the necroinflammatory activity grade were observed in this study. These results suggest that the co-occurrence of fibrosis and necroinflammation-but not that of fat or iron-renders the use of $\mathrm{R} 2{ }^{*}$ problematic for characterizing liver fibrosis in patients with CLDs. This could explain why QSM combined with R2* as the biomarker showed a decreased diagnostic performance compared to QSM alone.

Chronic hepatitis B patients represented a significant part of our study sample. Approximately one-third of cirrhosis cases worldwide are caused by the hepatitis B virus (HBV) infection (50), and liver fibrosis may develop to cirrhosis. As the current gold standard of liver fibrosis, invasive liver biopsy is a risky procedure, especially when HBV can spread through blood. The drawbacks, which include patient reluctance, pain, and potential complications, also cannot be ignored. Therefore, by enabling accurate diagnosis of the degree of liver fibrosis, QSM may potentially help physicians better manage chronic hepatitis $B$ patients and make informed clinical decisions.

This study has some limitations. First, chronic hepatitis B patients represented a significant part of our study.
Inhomogeneous distribution of CLD etiology may also have an effect on the performance of the MRI measurements. A further study should be performed on a larger number of CLD patients with varying fibrosis stages and etiologies. Second, the liver biopsy specimen had a $1 \mathrm{~mm}$ diameter and $17 \mathrm{~mm}$ length, which may not be representative of the whole liver tissue. Hepatic fibrosis, necroinflammatory activity, iron load, and steatosis in this specimen may not be representative of the whole liver because the development of CLDs is very heterogeneous. Third, the cutoff values were determined within a small sample. Further studies with larger cohorts are needed to evaluate and validate clinically efficient cutoff values. Fourth, the quantitative MR images including QSM, R2*, and PDFF were generated under a breath-hold sequence, which tended to have low signalto-noise ratio (SNR) and was prone to respiratory artifacts due to the relatively long breath-hold duration of $17 \mathrm{~s}$. The acquisition time could be shortened by reducing the number of slices and including only the central liver, but this would also decrease the SNR. Reducing the spatial resolution could shorten the acquisition time and improve SNR, but the pixel size would be decreased. Both the low coverage and low resolution would lead to loss of contrast and errors in susceptibility maps $(51,52)$. In future work, a navigator motion compensation approach (53) may be used for free-breathing data acquisition in liver QSM to produce consistently good quality maps. Fifth, liver QSM acquisition can be optimized by including in-phase echoes (25). The top or bottom of the liver may have more artifacts because a large variation of magnetic susceptibility and background magnetic field exists in these regions and may affect the reliability of the susceptibility measurement. The preconditioning method can be used to deal with the presence of air in the stomach and intestines of large susceptibility contrasts from the liver (54). With these data acquisition and reconstruction improvements, liver QSM quality may be as robust as brain QSM (19) and suitable for wide dissemination in clinical studies (23). Sixth, the effects of various physiological and technical factors on the susceptibility measurement may still need further assessment.

In conclusion, a significant decrease in liver susceptibility is associated with a corresponding progression in hepatic fibrosis. Liver susceptibility may be a promising marker for assessing significant fibrosis. With further study, QSM can potentially be used as an alternative tool for the noninvasive detection and diagnosis of the severity of hepatic fibrosis in patients with CLDs. 


\section{Acknowledgments}

Funding: This work was supported by the National Natural Science Foundation of China (grant numbers 81271533, 81730109); the Siming Scholar Foundation of Shuguang Hospital Affiliated to Shanghai University of Traditional Chinese Medicine (grant number SGXZ-201902); the National Science \& Technology Major Project (grant number 2018ZX10302204); and the Shanghai "Rising Stars of Medical Talent" Youth Development Program Youth Medical Talents - Medical Imaging Practitioner Program (grant number SHWRS(2020)-087).

\section{Footnote}

Conflicts of Interest: All authors have completed the ICMJE uniform disclosure form (available at http:// dx.doi.org/10.21037/qims-20-720). CF and XY, from the commercial company Siemens Healthcare, were MR collaborating scientists providing technical support under the Siemens collaboration regulations and had no financial or other conflicts with respect to this study. The other authors have no conflicts of interest to declare.

Ethical Statement: This study was conducted after obtaining approval from the Institutional Review Board of Shuguang Hospital affiliated with Shanghai University of Traditional Chinese Medicine (approval number: 2018-625-54-01). Written informed consent was obtained from the patients for publication of this study and any accompanying images. A copy of the written consent is available for review by the editor-in-chief of this journal.

Open Access Statement: This is an Open Access article distributed in accordance with the Creative Commons Attribution-NonCommercial-NoDerivs 4.0 International License (CC BY-NC-ND 4.0), which permits the noncommercial replication and distribution of the article with the strict proviso that no changes or edits are made and the original work is properly cited (including links to both the formal publication through the relevant DOI and the license). See: https://creativecommons.org/licenses/by-nc-nd/4.0/.

\section{References}

1. Bataller R, Brenner DA. Liver fibrosis. J Clin Invest 2005;115:209-18.

2. Fuchs BC, Hoshida Y, Fujii T, Wei L, Yamada S, Lauwers
GY, McGinn CM, DePeralta DK, Chen X, Kuroda T, Lanuti M, Schmitt AD, Gupta S, Crenshaw A, Onofrio R, Taylor B, Winckler W, Bardeesy N, Caravan P, Golub TR, Tanabe KK. Epidermal growth factor receptor inhibition attenuates liver fibrosis and development of hepatocellular carcinoma. Hepatology 2014;59:1577-90.

3. Rockey DC, Bissell DM. Noninvasive measures of liver fibrosis. Hepatology 2006;43:S113-20.

4. Annet L, Peeters F, Abarca-Quinones J, Leclercq I, Moulin P, Van Beers BE. Assessment of diffusionweighted MR imaging in liver fibrosis. J Magn Reson Imaging 2007;25:122-8.

5. Hagiwara M, Rusinek H, Lee VS, Losada M, Bannan MA, Krinsky GA, Taouli B. Advanced liver fibrosis: Diagnosis with 3D whole-liver perfusion MR imaging Initial experience. Radiology 2008;246:926-34.

6. Chen BB, Hsu CY, Yu CW, Wei SY, Kao JH, Lee HS, Shih TT. Dynamic contrast-enhanced magnetic resonance imaging with Gd-EOB-DTPA for the evaluation of liver fibrosis in chronic hepatitis patients. Eur Radiol 2012;22:171-80.

7. Allkemper T, Sagmeister F, Cicinnati V, Beckebaum S, Kooijman H, Kanthak C, Stehling C, Heindel W. Evaluation of fibrotic liver disease with wholeliver T1rho MR imaging: a feasibility study at $1.5 \mathrm{~T}$. Radiology 2014;271:408-15.

8. Zhao F, Zhou N, Wang JL, Zhou H, Zou LQ, Zhong WX, He J, Zheng CJ, Yan SX, Wáng YXJ. Collagen deposition in the liver is strongly and positively associated with T1rho elongation while fat deposition is associated with T1rho shortening: an experimental study of methionine and choline-deficient (MCD) diet rat model. Quant Imaging Med Surg 2020;10:2307-21.

9. Balassy C, Feier D, Peck-Radosavljevic M, Wrba F, Witoszynskyj S, Kiefer B, Reiter G, Dai Y, BaSsalamah A. Susceptibility-weighted MR imaging in the grading of liver fibrosis: a feasibility study. Radiology 2014:270:149-58.

10. Venkatesh SK, Yin M, Takahashi N, Glockner JF, Talwalkar JA, Ehman RL. Non-invasive detection of liver fibrosis: MR imaging features vs. MR elastography. Abdom Imaging 2015;40:766-75.

11. Gallego-Durán R, Cerro-Salido P, Gomez-Gonzalez E, Pareja MJ, Ampuero J, Rico MC, Aznar R, VilarGomez E, Bugianesi E, Crespo J, González-Sánchez FJ, Aparcero R, Moreno I, Soto S, Arias-Loste MT, Abad J, Ranchal I, Andrade RJ, Calleja JL, Pastrana M, Iacono OL, Romero-Gómez M. Imaging biomarkers for 
steatohepatitis and fibrosis detection in non-alcoholic fatty liver disease. Sci Rep 2016;6:31421.

12. Pavlides M, Banerjee R, Tunnicliffe EM, Kelly C, Collier J, Wang LM, Fleming KA, Cobbold JF, Robson MD, Neubauer S, Barnes E. Multiparametric magnetic resonance imaging for the assessment of non-alcoholic fatty liver disease severity. Liver Int 2017;37:1065-73.

13. Petitclerc L, Sebastiani G, Gilbert G, Cloutier G, Tang A. Liver fibrosis: Review of current imaging and MRI quantification techniques. J Magn Reson Imaging 2017;45:1276-95.

14. de Rochefort L, Liu T, Kressler B, Liu J, Spincemaille P, Lebon V, Wu J, Wang Y. Quantitative susceptibility map reconstruction from $M R$ phase data using bayesian regularization: validation and application to brain imaging. Magn Reson Med 2010;63:194-206.

15. Dong J, Liu T, Chen F, Zhou D, Dimov A, Raj A, Cheng Q, Spincemaille P, Wang Y. Simultaneous phase unwrapping and removal of chemical shift (SPURS) using graph cuts: application in quantitative susceptibility mapping. IEEE Trans Med Imaging 2015;34:531-40.

16. Wang Y, Liu T. Quantitative susceptibility mapping (QSM): Decoding MRI data for a tissue magnetic biomarker. Magn Reson Med 2015;73:82-101.

17. Dimov AV, Liu T, Spincemaille P, Ecanow JS, Tan H, Edelman RR, Wang Y. Joint estimation of chemical shift and quantitative susceptibility mapping (chemical QSM). Magn Reson Med 2015;73:2100-10.

18. Sharma SD, Hernando D, Horng DE, Reeder SB. Quantitative susceptibility mapping in the abdomen as an imaging biomarker of hepatic iron overload. Magn Reson Med 2015;74:673-83.

19. Deh K, Nguyen TD, Eskreis-Winkler S, Prince MR, Spincemaille P, Gauthier S, Kovanlikaya I, Zhang Y, Wang Y. Reproducibility of quantitative susceptibility mapping in the brain at two field strengths from two vendors. J Magn Reson Imaging 2015;42:1592-600.

20. Hinoda T, Fushimi Y, Okada T, Fujimoto K, Liu C, Yamamoto A, Okada T, Kido A, Togashi K. Quantitative Susceptibility Mapping at $3 \mathrm{~T}$ and $1.5 \mathrm{~T}$ : Evaluation of Consistency and Reproducibility. Invest Radiol 2015;50:522-30.

21. Lin PY, Chao TC, Wu ML. Quantitative susceptibility mapping of human brain at 3T: a multisite reproducibility study. AJNR Am J Neuroradiol 2015;36:467-74.

22. Santin MD, Didier M, Valabregue R, Yahia Cherif L, Garcia-Lorenzo D, Loureiro de Sousa P, Bardinet E, Lehericy S. Reproducibility of R2 * and quantitative susceptibility mapping (QSM) reconstruction methods in the basal ganglia of healthy subjects. NMR Biomed 2017;30. doi: 10.1002/nbm.3491.

23. Spincemaille P, Liu Z, Zhang S, Kovanlikaya I, Ippoliti M, Makowski M, Watts R, de Rochefort L, Venkatraman V, Desmond P, Santin MD, Lehericy S, Kopell BH, Peran $\mathrm{P}$, Wang Y. Clinical Integration of Automated Processing for Brain Quantitative Susceptibility Mapping: MultiSite Reproducibility and Single-Site Robustness. J Neuroimaging 2019;29:689-98.

24. Zhang X, Guo Y, Chen Y, Mei Y, Chen J, Wang J, Feng Y, Zhang X. Reproducibility of quantitative susceptibility mapping in lumbar vertebra. Quant Imaging Med Surg 2019;9:691-9.

25. Jafari R, Sheth S, Spincemaille P, Nguyen TD, Prince MR, Wen Y, Guo Y, Deh K, Liu Z, Margolis D, Brittenham GM, Kierans AS, Wang Y. Rapid automated liver quantitative susceptibility mapping. J Magn Reson Imaging 2019;50:725-32.

26. Wáng YXJ, Wang X, Wu P, Wang Y, Chen W, Chen $\mathrm{H}, \mathrm{Li}$ J. Topics on quantitative liver magnetic resonance imaging. Quant Imaging Med Surg 2019;9:1840-90.

27. Luo J, He X, d'Avignon DA, Ackerman JJ, Yablonskiy DA. Protein-induced water $1 \mathrm{H}$ MR frequency shifts: contributions from magnetic susceptibility and exchange effects. J Magn Reson 2010;202:102-8.

28. Li JQ, Lin HM, Liu T, Zhang ZW, Prince MR, Gillen K, Yan X, Song Q, Hua T, Zhao XC, Zhang M, Zhao Y, Li GY, Tang GY, Yang G, Brittenham GM, Wang Y. Quantitative susceptibility mapping (QSM) minimizes interference from cellular pathology in R2(star) estimation of liver iron concentration. J Magn Reson Imaging 2018;48:1069-79.

29. Dong JW, Liu T, Chen F, Zhou D, Dimov A, Raj A, Cheng Q, Spincemaille P, Wang Y. Simultaneous phase unwrapping and removal of chemical shift (SPURS) using graph cuts: application in quantitative susceptibility mapping. IEEE Trans Med Imaging 2015;34:531-40.

30. Yu H, McKenzie CA, Shimakawa A, Vu AT, Brau AC, Beatty PJ, Pineda AR, Brittain JH, Reeder SB. Multiecho reconstruction for simultaneous waterfat decomposition and $\mathrm{T}^{*}$ estimation. J Magn Reson Imaging 2007;26:1153-61.

31. Liu T, Khalidov I, de Rochefort L, Spincemaille P, Liu J, Tsiouris AJ, Wang Y. A novel background field removal method for MRI using projection onto dipole fields (PDF). NMR Biomed 2011;24:1129-36.

32. Liu J, Liu T, de Rochefort L, Ledoux J, Khalidov I, Chen 
WW, Tsiouris AJ, Wisnieff C, Spincemaille P, Prince MR, Wang Y. Morphology enabled dipole inversion for quantitative susceptibility mapping using structural consistency between the magnitude image and the susceptibility map. Neuroimage 2012;59:2560-8.

33. Lin H, Wei H, He N, Fu C, Cheng S, Shen J, Wang B, Yan X, Liu C, Yan F. Quantitative susceptibility mapping in combination with water-fat separation for simultaneous liver iron and fat fraction quantification. Eur Radiol 2018;28:3494-504.

34. Ishak K, Baptista A, Bianchi L, Callea F, De Groote J, Gudat F, Denk H, Desmet V, Korb G, MacSween RN, Phillips MJ, Portmann BG, Poulsen H, Scheuer PJ, Schmid M, Thaler H. Histological grading and staging of chronic hepatitis. J Hepatol 1995;22:696-9.

35. Terrault NA, Lok ASF, McMahon BJ, Chang KM, Hwang JP, Jonas MM, Brown RS Jr, Bzowej NH, Wong JB. Update on prevention, diagnosis, and treatment of chronic hepatitis B: AASLD 2018 Hepatitis B Guidance. Clin Liver Dis (Hoboken) 2018;12:33-4.

36. Liver EAftSot. EASL 2017 Clinical Practice Guidelines on the management of hepatitis B virus infection. J Hepatol 2017;67:370-98.

37. Bedossa P, Poynard T. An algorithm for the grading of activity in chronic hepatitis C. The METAVIR Cooperative Study Group. Hepatology 1996;24:289-93.

38. Kleiner DE, Brunt EM, Van Natta M, Behling C, Contos MJ, Cummings OW, Ferrell LD, Liu YC, Torbenson MS, Unalp-Arida A, Yeh M, McCullough AJ, Sanyal AJ, Nonalcoholic Steatohepatitis Clinical Research N. Design and validation of a histological scoring system for nonalcoholic fatty liver disease. Hepatology 2005;41:1313-21.

39. Vali Y, Lee J, Boursier J, Spijker R, Loffler J, Verheij J, Brosnan MJ, Bocskei Z, Anstee QM, Bossuyt PM, Zafarmand MH, Team LSR. Enhanced liver fibrosis test for the non-invasive diagnosis of fibrosis in patients with NAFLD: A systematic review and meta-analysis. J Hepatol 2020;73:252-62.

40. Xu XY, Wang WS, Zhang QM, Li JL, Sun JB, Qin TT, Liu HB. Performance of common imaging techniques vs serum biomarkers in assessing fibrosis in patients with chronic hepatitis B: A systematic review and metaanalysis. World J Clin Cases 2019;7:2022-37.

41. Reiter R, Tzschatzsch H, Schwahofer F, Haas M, Bayerl C, Muche M, Klatt D, Majumdar S, Uyanik M, Hamm B, Braun J, Sack I, Asbach P. Diagnostic performance of tomoelastography of the liver and spleen for staging hepatic fibrosis. Eur Radiol 2020;30:1719-29.

42. Schawkat K, Ciritsis A, von Ulmenstein S, HoncharovaBiletska H, J?ngst C, Weber A, Gubler C, Mertens J, Reiner CS. Diagnostic accuracy of texture analysis and machine learning for quantification of liver fibrosis in MRI: correlation with MR elastography and histopathology. Eur Radiol 2020;30:4675-85.

43. Ajmera VH, Liu A, Singh S, Yachoa G, Ramey M, Bhargava M, Zamani A, Lopez S, Mangla N, Bettencourt R, Rizo E, Valasek M, Behling C, Richards L, Sirlin C, Loomba R. Clinical utility of an increase in magnetic resonance elastography in predicting fibrosis progression in nonalcoholic fatty liver disease. Hepatology 2020;71:849-60.

44. Wei H, Gibbs E, Zhao P, Wang N, Cofer GP, Zhang Y, Johnson GA, Liu C. Susceptibility tensor imaging and tractography of collagen fibrils in the articular cartilage. Magn Reson Med 2017;78:1683-90.

45. Wei H, Decker K, Nguyen H, Cao S, Tsai TY, Dianne Guy C, Bashir M, Liu C. Imaging diamagnetic susceptibility of collagen in hepatic fibrosis using susceptibility tensor imaging. Magn Reson Med 2020;83:1322-30.

46. Seto WK, Hui RWH, Mak LY, Fung J, Cheung KS, Liu KSH, Wong DKH, Lai CL, Yuen MF. Association between hepatic steatosis, measured by controlled attenuation parameter, and fibrosis burden in chronic hepatitis B. Clin Gastroenterol Hepatol 2018;16:575-583.e2.

47. Powell EE, Jonsson JR, Clouston AD. Steatosis: co-factor in other liver diseases. Hepatology 2005;42:5-13.

48. Sharma P, Altbach M, Galons JP, Kalb B, Martin DR. Measurement of liver fat fraction and iron with MRI and MR spectroscopy techniques. Diagn Interv Radiol 2014;20:17-26.

49. Kershenobich Stalnikowitz D, Weissbrod AB. Liver fibrosis and inflammation. A review. Ann Hepatol 2003;2:159-63.

50. Kawada N. Evolution of hepatic fibrosis research. Hepatol Res 2011;41:199-208.

51. Karsa A, Punwani S, Shmueli K. The effect of low resolution and coverage on the accuracy of susceptibility mapping. Magn Reson Med 2019;81:1833-48.

52. Zhou D, Cho JH, Zhang JW, Spincemaille P, Wang Y. Susceptibility underestimation in a high-susceptibility phantom: Dependence on imaging resolution, magnitude contrast, and other parameters. Magn Reson Med 2017;78:1080-6.

53. Wang Y, Rossman PJ, Grimm RC, Wilman AH, 
Riederer SJ, Ehman RL. 3D MR angiography of pulmonary arteries using real-time navigator gating and magnetization preparation. Magn Reson Med 1996;36:579-87.

Cite this article as: Qu Z, Yang S, Xing F, Tong R, Yang C, Guo R, Huang J, Lu F, Fu C, Yan X, Hectors S, Gillen K, Wang Y, Liu C, Zhan S, Li J. Magnetic resonance quantitative susceptibility mapping in the evaluation of hepatic fibrosis in chronic liver disease: a feasibility study. Quant Imaging Med Surg 2021;11(4):1170-1183. doi: 10.21037/qims-20-720
54. Liu Z, Kee Y, Zhou D, Wang Y, Spincemaille P. Preconditioned total field inversion (TFI) method for quantitative susceptibility mapping. Magn Reson Med 2017;78:303-15. 